Check for updates

Cite this: RSC Adv., 2019, 9, 20216

Received 12th April 2019

Accepted 4th June 2019

DOI: 10.1039/c9ra02784j

rsc.li/rsc-advances

\section{Fly ash based robust biocatalyst generation: a sustainable strategy towards enhanced green biosurfactant production and waste utilization}

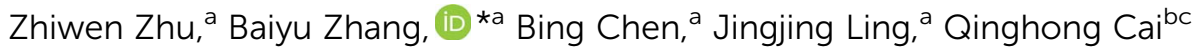 \\ and Tahir Husain ${ }^{a}$
}

Biosurfactants have been well recognized as an environmentally friendly alternative to chemical surfactants. However, their production remains challenging due to low productivity, short-term microbe stability and the potentially toxic by-products generated in the growth media. To overcome these challenges, the emerging biofilm-based biosynthesis was investigated in this study. A fresh insight into the biosynthesis process was provided through using waste fly ash as a carrier material. The biofilm produced by biosurfactant producer $B$. subtilis N3-1P attached onto the surface of fly ash acted as a robust and effective biocatalyst. Zeta potential analysis and scanning electron microscope (SEM) characterization were conducted to help unravel the biocatalyst formation. High-value biosurfactant products were then produced in an efficient and sustainable manner. Stimulation by a fly ash assisted biocatalyst on biosurfactant production was confirmed. The biosurfactant yield was boosted over ten times after 24 hours, at a fly ash dosage of $0.5 \%$. The highest biosurfactant yield was achieved after five days, with a final productivity of 305 critical micelle dilution. The underlying mechanism of fly ash assisted biosurfactant production was tracked through it exerting an effect on the quorum sensing system. Fourier-transform infrared (FTIR) spectroscopy and matrix assisted laser desorption/ionization-time of flight (MALDI-TOF) analysis demonstrated that the final biosurfactant product belonged to the lipopeptides. This research output is expected to accelerate the development of more effective bioreactors, and make a continuous contribution to high-value product generation and waste reduction.

\section{Introduction}

Surface active agents, such as lipopeptides, glycolipids, phospholipids, fatty acids, and neutral lipids, if produced by microorganisms during their growth, are named biosurfactants. ${ }^{1}$ Compared to chemically synthetic surfactants, biosurfactants offer the advantages of retaining a stable and effective performance even under extreme environmental conditions, and they cause little or no environmental impact due to their low toxicity and high biodegradability. ${ }^{2,3}$ They exhibit high surface activities and low critical micelle concentrations (CMCs) and have, therefore, attracted a lot of attention in recent years. ${ }^{4}$ As an environmentally friendly alternative to chemical surfactants, biosurfactants could serve as detergents, emulsifiers, and foaming, wetting, and dispersing agents in

${ }^{a}$ NRPOP Laboratory, Faculty of Engineering and Applied Science, Memorial University, St.John's, NL, A1B 3X5, Canada.E-mail: bzhang@mun.cab

${ }^{b}$ Biotechnology Research Institute of the National Research Council of Canada, 6100 Royalmount Ave, Montreal, QC H4P 2R2, Canada

'Department of Natural Resource Sciences, Faculty of Agricultural and Environmental Sciences, McGill University, Macdonald Campus, 21111 Lakeshore Rd, Ste Anne-deBellevue, QC, H9X 3V9, Canada environmental, oil and pharmaceutical industries. ${ }^{5}$ Despite their environmentally favorable characteristics, the economic feasibility of biosurfactants remains problematic owing to their poor production rate, arising primarily from the complex regulation system during fermentation and limited number of effective production cells. ${ }^{6}$

Zhi et $a .^{7}$ indicated that biosurfactant producers such as Bacillus subtilis can generate surfactin, a lipopeptide, through biosynthetic regulation of a quorum sensing system. In this system, surfactin synthesis, competence development and sporulation are cross-linked within a complex network of pheromones and pleiotropic regulators. As a consequence of quorum sensing, surfactin synthesis is dependent on cell density, preventing constant production and limiting overall yields. Methodologies for increasing cell density and thus enhancing biosurfactant productivity are important.

Biofilm is an assemblage of microorganisms embedded in a self-produced matrix of extracellular polymeric substances (EPSs). ${ }^{8}$ They can self-immobilize and self-regenerate on all kinds of interfaces with well-organized metabolism and, in the meantime, offer protection for the microbes inside. ${ }^{9}$ Given that whole-cell mobilization has been suggested as an effective approach to reduce fermentation time, ${ }^{\mathbf{1 0}}$ the robust and long- 
lasting features of biofilms make them attractive as biocatalysts for organic synthesis with high productivity, particularly when cell viability is affected by substrates and/or metabolites. ${ }^{\mathbf{1 1 , 1 2}}$ The proper selection of a solid carrier can greatly improve the growth rate of biofilm, and thereby effectively increase the density of cells and stimulate the production of target metabolites. ${ }^{13}$ Recent findings have indicated that harnessing porous solid carriers with larger surface areas, such as activated carbon and expanded clay, could promote gas exchange, and provide larger cell attachment and more immobilization sites for microbes. ${ }^{6,14}$ Solid carriers could also provide a larger buffer capacity under extreme culturing conditions and hence protect the microbes from biotic and abiotic stresses. ${ }^{6,13}$

Fly ash (FA) is a municipal solid waste produced worldwide due to the combustion of coal at high temperature. Owing to the concentrated toxic heavy metals in the ash, FA is regarded as a hazardous waste. Research has centered on the treatment of FA through detoxification and potential resource recovery. For example, treated FA can be beneficially used as a natural absorbent after proper treatment, given its porous structure. However, no attempt has ever been made to use FA as a solid carrier for microbial growth.

Therefore, in this work, FA was tried as a solid carrier for facilitating the cost-effective and highly efficient biosynthesis of biosurfactants for the first time. Two hypotheses regarding the role of FA on the biosynthesis process were examined: (1) the porous structure of FA could provide a larger surface area for the attachment of biocatalyst, thereby greatly stimulating biosurfactant production, and (2) the immobilized bacterial biofilm may have a positive effect on the detoxification of FA by means of a bioleaching process. The biosurfactant-producing microorganism applied was $B$. subtilis $\mathrm{N} 3-1 \mathrm{P}$, which was isolated from the Atlantic Ocean. The performance of FA on cell growth and biosurfactant production was investigated. The effect of FA dosage on biosurfactant production was examined using parameters including surface tension (ST), emulsification activity, and solution dilution as responses. The generated biosurfactant product was characterized by determining ST and CMC. Its structure was further characterized using Fourier-transform infrared (FTIR) spectroscopy and matrix assisted laser desorption/ionization time of flight mass spectrometry (MALDI-TOF MS).

\section{Materials and methods}

\subsection{FA as solid carrier}

The FA to be used as a platform for biosurfactant production was obtained from the Corner Brook Pulp and Paper (CBPP) plants in Newfoundland and Labrador, Canada. Bunker C oil had been mixed with wasted pulpwood as a fuel during the thermal mechanical pulp process. Generated fly ash was then collected from the power boiler and subjected to air drying. The properties of the fly ash are listed in Table 1. FA was characterized before and after incubation with FTIR by the KBr pellet method and scanning electron microscopy (SEM).
Table 1 Characteristics of CBPP FA

\begin{tabular}{ll}
$\mathrm{pH}$ & 12 \\
Density $\left(\mathrm{g} \mathrm{cm}^{-3}\right)$ & 0.45 \\
Moisture content $(\%)$ & 0.89 \\
Surface area $\left(\mathrm{m}^{2} \mathrm{~g}^{-1}\right)$ & 249.4 \\
$\mathrm{C} / \mathrm{N}$ ratio & 572.95 \\
\hline Elemental content in solid (unit: $\left.\mathrm{mg} \mathrm{kg}^{-1}\right)$ & \\
\hline $\mathrm{Mg}$ & \\
$\mathrm{Al}$ & 511.65 \\
$\mathrm{Fe}$ & 947.03 \\
$\mathrm{P}$ & 784.20 \\
$\mathrm{Cl}$ & 114.33 \\
$\mathrm{Zn}$ & 11634 \\
$\mathrm{Cu}$ & 11.72 \\
$\mathrm{~Pb}$ & 7.28 \\
$\mathrm{~V}$ & 2.25 \\
$\mathrm{Cr}$ & 15.46 \\
$\mathrm{Ni}$ & 4.73 \\
$\mathrm{Ca}$ & 15.96 \\
\end{tabular}

\subsection{Biosurfactant-producing microorganisms}

B. subtilis is a motile, Gram-positive, rod-shaped endosporeforming bacterium widely studied in biofilm formation. It is famous for producing biosurfactants, especially effective lipopeptide biosurfactants. The bacterium used in this study, $B$. subtilis $\mathrm{N} 3-1 \mathrm{P}$, was screened from oily contaminated seawater samples. ${ }^{15}$ This strain was identified as a promising and economic biosurfactant producer among the screened bacteria, whose product possessed strong surface activity and high emulsification capacity.

The composition of the culture medium for $B$. subtilis N3-1P was as follows: BD Difco ${ }^{\mathrm{TM}}$ Marine Broth (Fisher Scientific, Canada) $37.4 \mathrm{~g}$ in $1 \mathrm{~L}$ of distilled water. A loopful of a bacteria colony was transferred into a $125 \mathrm{~mL}$ Erlenmeyer flask containing $50 \mathrm{~mL}$ of inoculum broth. This seeded culture medium was initially grown on a rotary incubator shaker (VWR, Canada) at $200 \mathrm{rpm}$ for $24 \mathrm{~h}$ at room temperature to reach its exponential growth phase. The biosurfactant production medium comprised ( $\left.\mathrm{g} \mathrm{L}^{-1}\right)$ : sucrose (30), $\mathrm{NH}_{4} \mathrm{NO}_{3}$ (10), $\mathrm{NaCl}$ (15), $\mathrm{KH}_{2} \mathrm{PO}_{4}$ (3.4), $\mathrm{K}_{2} \mathrm{HPO}_{4} \cdot 3 \mathrm{H}_{2} \mathrm{O}$ (4.4), $\mathrm{MgSO}_{4} \cdot 7 \mathrm{H}_{2} \mathrm{O}$ (1.02), and yeast extract $(0.5)$.

\subsection{Biosurfactant production with FA}

Effect of FA on biocatalyst immobilization. FA was added into the production medium (Section 2.2) at a 1\% level to assist the attachment of the biofilm-based biocatalyst and medium without FA was used as a control. A seeded culture medium (Section 2.2) was used as inoculum at 1\% (v/v) level. Samples were collected every six hours for a total of 24 hours. FA particles in the culture broth were removed through a filtration process. The filtrate containing culture broth was centrifuged at $12000 \mathrm{rpm}$ for $10 \mathrm{~min}$ to remove the remaining cells. Filtrated FA particles were gently washed three times with PBS buffer solution and then subjected to zeta potential measurement. The growth behavior of the biofilm-based biocatalyst was quantified 
a)

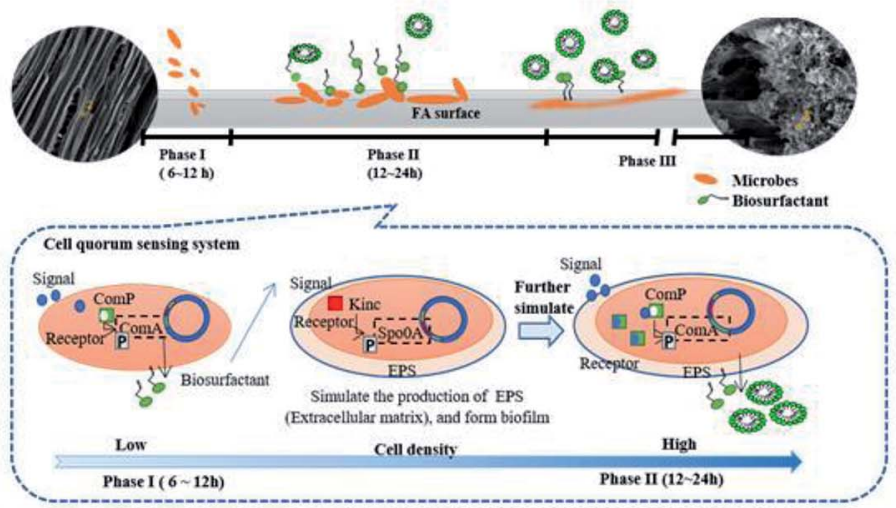

c)

b)
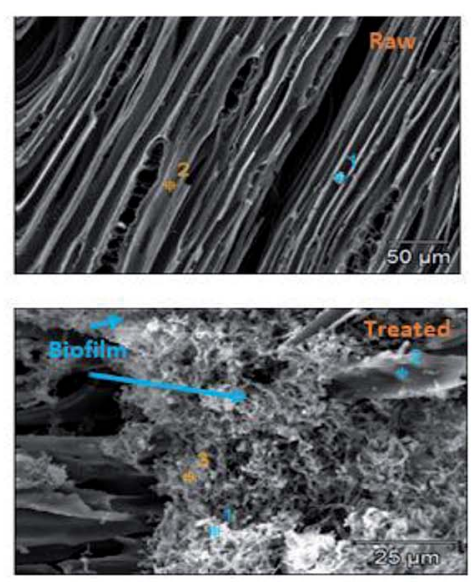
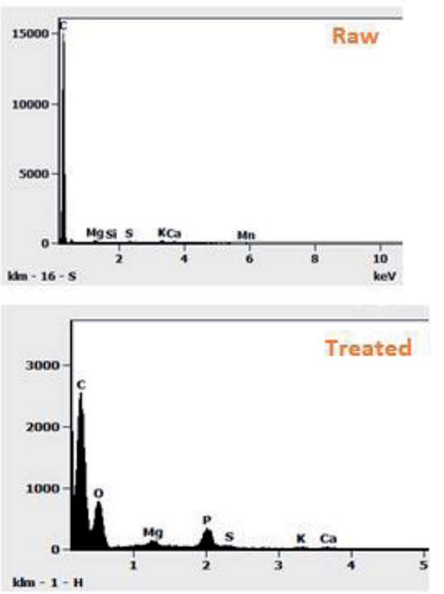
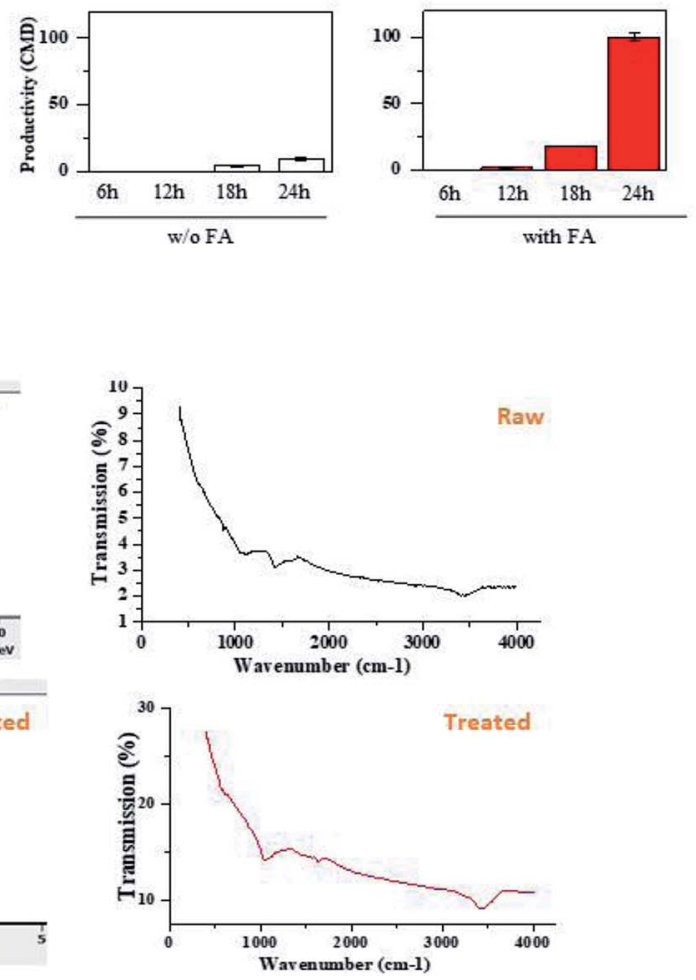

Fig. 1 (a) Mechanism of immobilized biocatalyst enhanced biosurfactant production on FA particles; (b) characterization of FA with SEM and FTIR; and (c) biosurfactant production with and without the existence of an FA-based platform.

by the variation in zeta potential. The immobilized biocatalyst on FA particles was further characterized with FTIR and SEM. All the samples were collected and analyzed in triplicate. The cell-free filtrate was analyzed for biosurfactant productivity using critical micelle dilution (CMD) as an indicator.

Effect of FA dosage on biosurfactant production. FA particles were added into the biosurfactant production medium (Section $2.2)$ at levels of $0.5 \%, 1 \%$ and $2 \%(\mathrm{w} / \mathrm{v})$. Medium without FA particles was used as a control. The seeded culture medium (Section 2.2) was used as inoculum at 1\% (v/v) level. Samples were collected at six-hour intervals for the first day and then every 24 hours for the next 6 days. Each sample was subjected to filtration to remove the FA particles and then centrifuged at $12000 \mathrm{rpm}$ for $10 \mathrm{~min}$ to remove the remaining cells. The fermentation process was monitored by measuring parameters such as ST, pH, and CMD. The FA particles were collected and further examined to determine the effect of biosurfactant adsorption on final productivity. The residual FA particles were treated to separate the adsorbed biosurfactant following the method described by Dubey et al. ${ }^{16}$ The optimum FA level and incubation time obtained from the above tests were selected for batch-scale biosurfactant production. All the analyses in this study were performed in triplicate.

\subsection{Characterization of the generated biosurfactant product}

The optimum FA addition level and incubation time derived from Section 2.2 were applied during batch-scale biosurfactant

Table 2 Zeta potential of FA as a function of incubation time at different dosages

\begin{tabular}{|c|c|c|c|c|c|c|}
\hline \multirow[b]{2}{*}{ Time (h) } & \multicolumn{2}{|l|}{$0.5 \% \mathrm{FA}$} & \multicolumn{2}{|l|}{$1 \%$ FA } & \multicolumn{2}{|l|}{$2 \% \mathrm{FA}$} \\
\hline & $(\xi)$ & SD (\%) & Zeta potential $(\xi)$ & $\mathrm{SD}(\%)$ & Zeta potential $(\xi)$ & $\mathrm{SD}(\%)$ \\
\hline 0 & -3.45 & 6 & -9.54 & 6 & -10.89 & 2 \\
\hline 6 & -13.78 & 5 & -15.05 & 5 & -13.43 & 5 \\
\hline 12 & -14.48 & 4 & -15.05 & 3 & -14.08 & 3 \\
\hline
\end{tabular}




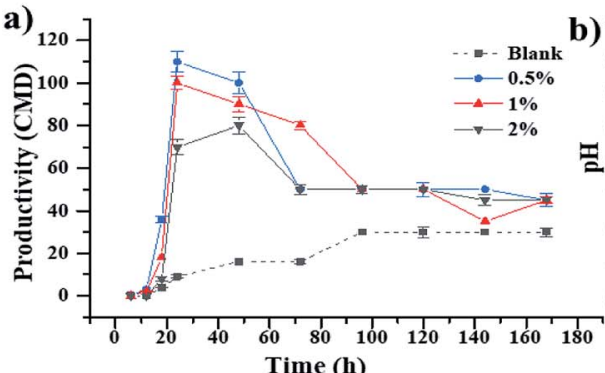

d)

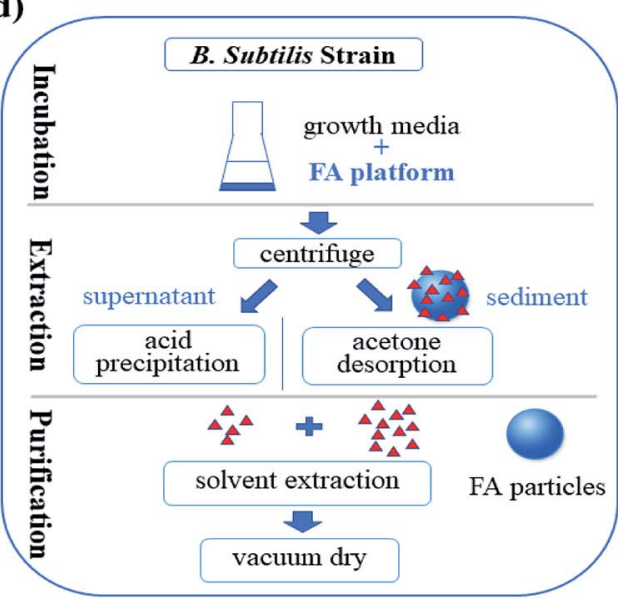

b) ${ }^{9.5}$. ......-B Blank

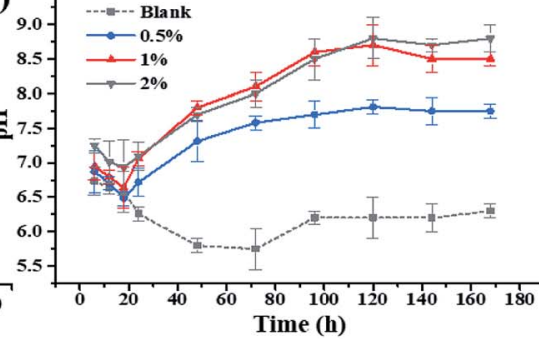

e)
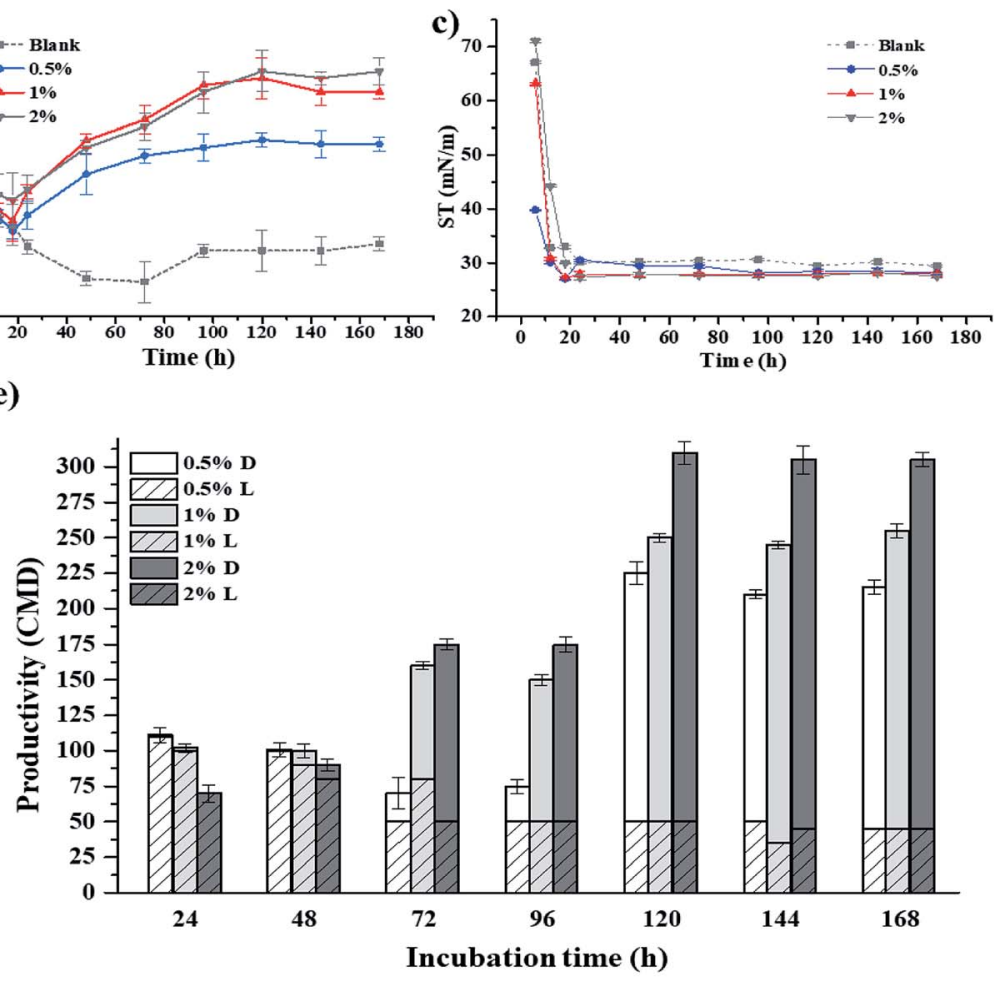

Fig. 2 Biosurfactant production with FA-based platform: (a) productivity of growth medium; (b) pH of growth medium; (c) ST of growth medium, (d) flow chart of the biosurfactant production process and (e) biosurfactant production in culture broth $(0.5 \% \mathrm{~L}, 1 \% \mathrm{~L}, 2 \% \mathrm{~L})$ and in FA particles $(0.5 \%$ D, $1 \%$ D, $2 \%$ D).

production. Biosurfactants in the FA particles were collected using the method described in Section 2.2. The FA-free culture broth was centrifuged at $12000 \mathrm{rpm}$ for $10 \mathrm{~min}$ to remove the remaining cells. Cell-free culture broth was extracted using an equal volume of chloroform-methanol $(1: 2 \mathrm{v} / \mathrm{v})$ solvent. The solvent was removed by rotary evaporation. Combined biosurfactant products were extracted from FA particles and culture medium, and then subjected to ST and CMC measurements. Their structures were further characterized using FTIR spectroscopy and MALDI-TOF-MS. All the characterization results were compared with those generated by the control.

\subsection{Bioleaching of heavy metals from FA}

Metal leachability from the FA particles during the biosurfactant production process was estimated. Cell-free culture mediums at a FA concentration of $1 \%$ and $2 \%$ collected from the process in Section 2.3 were further examined for their bioleaching behavior. Samples were collected and analyzed in duplicate, and a triplicate analysis was performed when the deviations were greater than $5 \%$. The concentrations of leached heavy metals were examined using ICP-MS. The differences between FA-based medium and control samples were recorded.

\subsection{Sample analysis}

pH and moisture content. The $\mathrm{pH}$ of FA from CBPP was measured following ASTM D1512-15b, and the moisture content was determined following ASTM D1512-05 (2012).
Surface tension (ST). A $15 \mathrm{~mL}$ cell-free culture medium was subjected to determination of ST in a Petri dish. ST was measured by the ring method, using a du Noüy tensiometer (CSC Scientific, USA). To ensure the reliability of the test results, an average of three independent measurements was taken.

CMC and CMD. CMC is defined as the minimum concentration of surfactant molecules to spontaneously form micelles. It was determined following the method described by Sheppard and Mulligan. ${ }^{17}$ The purified biosurfactant product from Section 2.4 was obtained and a series of biosurfactant solutions with different concentrations were prepared. The STs of the prepared solutions were measured. The CMC of the generated biosurfactant was determined by plotting the values of surface tension as a function of the associated biosurfactant concentration. The intercept of two straight lines extrapolated from the concentration-dependent and concentration-independent sections was defined as the CMC. ${ }^{17}$ CMD is an indicator of biosurfactant concentration in the medium. It corresponds to the dilution a medium requires to reach its $\mathrm{CMC} .{ }^{18}$ It was determined following the method described by Cai et al. ${ }^{19}$

Zeta potential. Determination of the FA zeta potential was modified from the methods described by Akgün ${ }^{20}$ and Li et al. ${ }^{21}$ FA samples collected from incubation samples during the first 24 hours were gently washed three times with 10 mM PBS buffer solution ( $\mathrm{pH}$ 7.4) and then dissolved into this PBS buffer solution to reach a final concentration of $1 \mathrm{mg} \mathrm{mL}^{-1}$. Each sample was gently shaken for 12 hours before measurement with a Malvern Zetasizer. 
<smiles>CC(C)CCCCCCCCCCC(CC(=O)NC(CCC(=O)O)C(=O)NC(CC(C)C)C(=O)NC(CC(C)C)C(=O)NC(C(=O)NC(CC(=O)O)C(=O)NC(CC(C)C)C(=O)NC(CC(C)C)C(=O)O)C(C)C)NC(=O)C(CC(C)C)NC(=O)C(C)C</smiles>

Surfactin

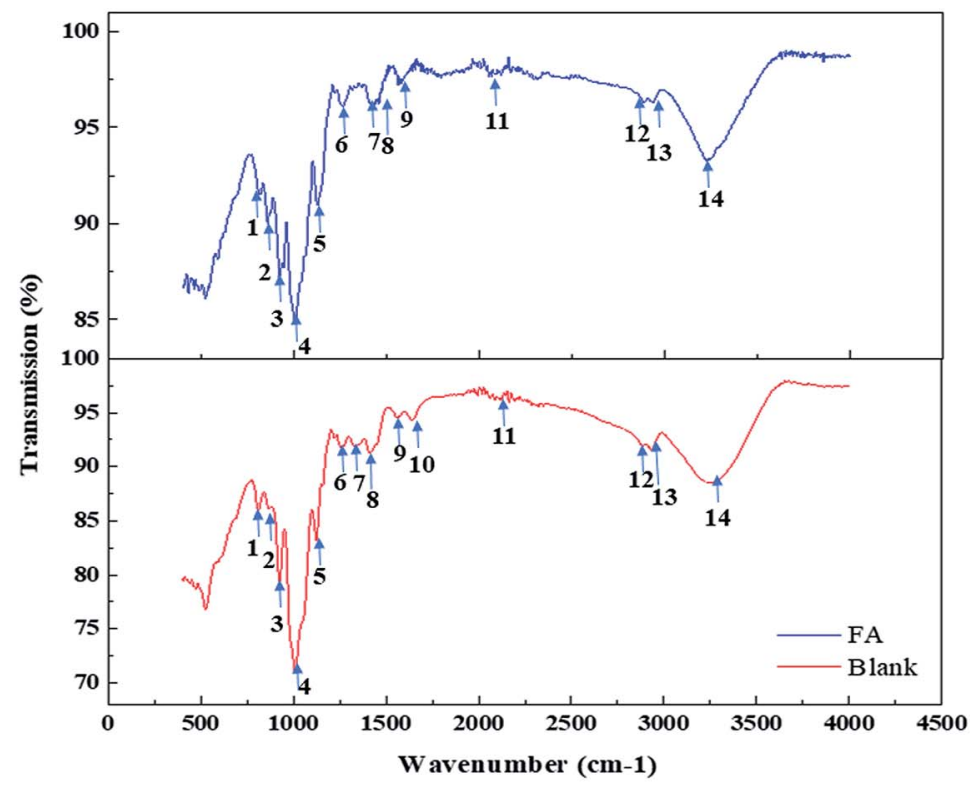

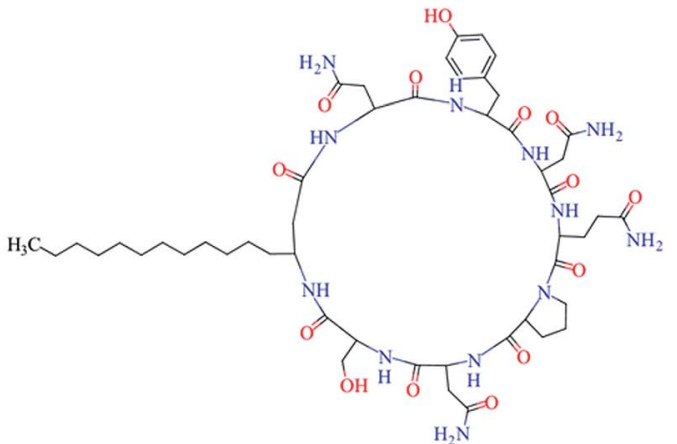

Iturin A

\begin{tabular}{|c|c|c|}
\hline Peak & Wave & Functional group \\
\hline 1 & 805.3 & $-\mathrm{CH}$ \\
\hline 2 & 856.67 & Aromatic $\mathrm{CH}(900-670$ (several)) \\
\hline 3 & 919.12 & $\begin{array}{l}\text { 1,3 Disubstitution (meta) }(810-750 \text { and } \\
900-860) \\
\text { 1,4-Disubstitution (para) }(860-800) \\
\text { Peroxides (-COOC-) }(890-820) \\
\text { Vinyl CH (995-985 and 915-890) }\end{array}$ \\
\hline 4 & 1001.85 & Primary amine, $\mathrm{CN}$ stretch \\
\hline 5 & 1128 & $\begin{array}{l}\text { Alkyl-substituted ether, (-CO) } \\
\text { Cyanate (-OCN and C-OCN stretch) }\end{array}$ \\
\hline 6 & 1256.85 & Primary or secondary $(-\mathrm{OH})$ \\
\hline 7 & 1421.86 & Vinyl $(-\mathrm{CH})$ \\
\hline 8 & 1446.54 & Methylene $\mathrm{CH}$ bend \\
\hline 9 & 1569.92 & Secondary amine, NH bend (R-NH-R) \\
\hline 10 & 1652.60 & $\begin{array}{l}\text { Alkenyl }(\mathrm{C}=\mathrm{C}) \text {; Secondary amine, } \mathrm{NH} \\
\text { bend; Open-chain imino }(-\mathrm{C}=\mathrm{N}-)\end{array}$ \\
\hline 11 & 2250 & $\begin{array}{l}\text { Cyanate }(-\mathrm{OCN} \text { and } \mathrm{C}-\mathrm{OCN} \text { stretch }) \\
\text { Isocyanate }(-\mathrm{N}=\mathrm{C}=\mathrm{O} \text { stretch })\end{array}$ \\
\hline 12 & 2880.97 & Methyl (-CH3) \\
\hline 13 & 2937.80 & Methylene (=CH-) \\
\hline 14 & 3234.66 & Hydroxyl (R-OH) \\
\hline
\end{tabular}

Fig. 3 FTIR analysis of biosurfactant produced by Bacillus strains with FA-based platform.

Trace metals. The trace metals in the FA samples were analyzed by the modified EPA Method 3050 using inductively coupled plasma mass spectrometry (ICP-MS, PerkinElmer ELAN DRCII, USA). A $100 \pm 10 \mathrm{mg}$ FA sample was weighed in a $15 \mathrm{~mL}$ Teflon vial with a screw cap. Then $3 \mathrm{~mL}$ of $8 \mathrm{~N}$ nitric acid $\left(\mathrm{HNO}_{3}\right)$ was added and heated on a hot plate at $70{ }^{\circ} \mathrm{C}$ for two days. The sample was cooled again. Afterwards, $1 \mathrm{~mL}$ of $\mathrm{HNO}_{3}$ and $1 \mathrm{~mL}$ of hydrogen peroxide $\left(\mathrm{H}_{2} \mathrm{O}_{2}\right)$ were added and the sample was heated at $70{ }^{\circ} \mathrm{C}$ for two days to remove organic matter. The sample was then dried and cooled. An additional $2 \mathrm{~mL}$ of $8 \mathrm{~N}$ $\mathrm{HNO}_{3}$ and $1 \mathrm{~mL}$ hydrofluoric acid (HF) were added to the sample and heated at $70{ }^{\circ} \mathrm{C}$ for two days. After drying and cooling, $3 \mathrm{~mL}$ of aqua regia $\left(\mathrm{v}_{\mathrm{HCl}}: \mathrm{v}_{\mathrm{HNO}_{3}}=3: 1\right)$ was added to the sample and heated at $70{ }^{\circ} \mathrm{C}$ for one day. The sample was eventually dried, cooled, and dissolved in $2 \% \mathrm{HNO}_{3}$. The solution was then diluted and analyzed by ICP-MS.

Fourier-transform infrared (FTIR) analysis. Both FA particles and biosurfactant products were examined with FTIR (Bruker Tensor). FA particles were characterized with the KBr-pellet method. Spectral measurements were performed in transmittance mode. Crude biosurfactant products were directly characterized with attenuated total reflection (ATR)-FTIR spectroscopy in the absorbance mode. IR was traced over the range of $400-4000 \mathrm{~cm}^{-1}$. All data were corrected for background.

MALDI-TOF mass spectra analysis. Biosurfactant products in filtrate, FA, and blank samples were examined with MALDI-TOF mass spectra by a SCIEX MALDI TOF/TOF System. Each purified biosurfactant sample was dissolved in $10 \mathrm{~mL}$ of distilled water and then passed through a $0.2 \mu \mathrm{m}$ filter before testing. For mass spectrometric analysis of isolated lipopeptide biosurfactants, a $2 \mu \mathrm{l}$ portion of biosurfactant solution was mixed with an equal volume of matrix medium (a saturated solution of $\alpha$-cyano-4hydroxycinnamic acid in 50\% aqueous acetonitrile containing $0.1 \%(\mathrm{v} / \mathrm{v})$ trifluoroacetic acid). Positive-ion detection and reflector mode were used. The acceleration and reflector voltages were 20 and $23.4 \mathrm{kV}$ in the pulsed ion extraction mode. Post-source decay (PSD) mass spectra were obtained using the same sample.

\section{Results and discussion}

\subsection{Effect of biocatalyst immobilization on microbe growth}

Previous studies have proved cell growth stimulation and product promotion through using certain types of porous 

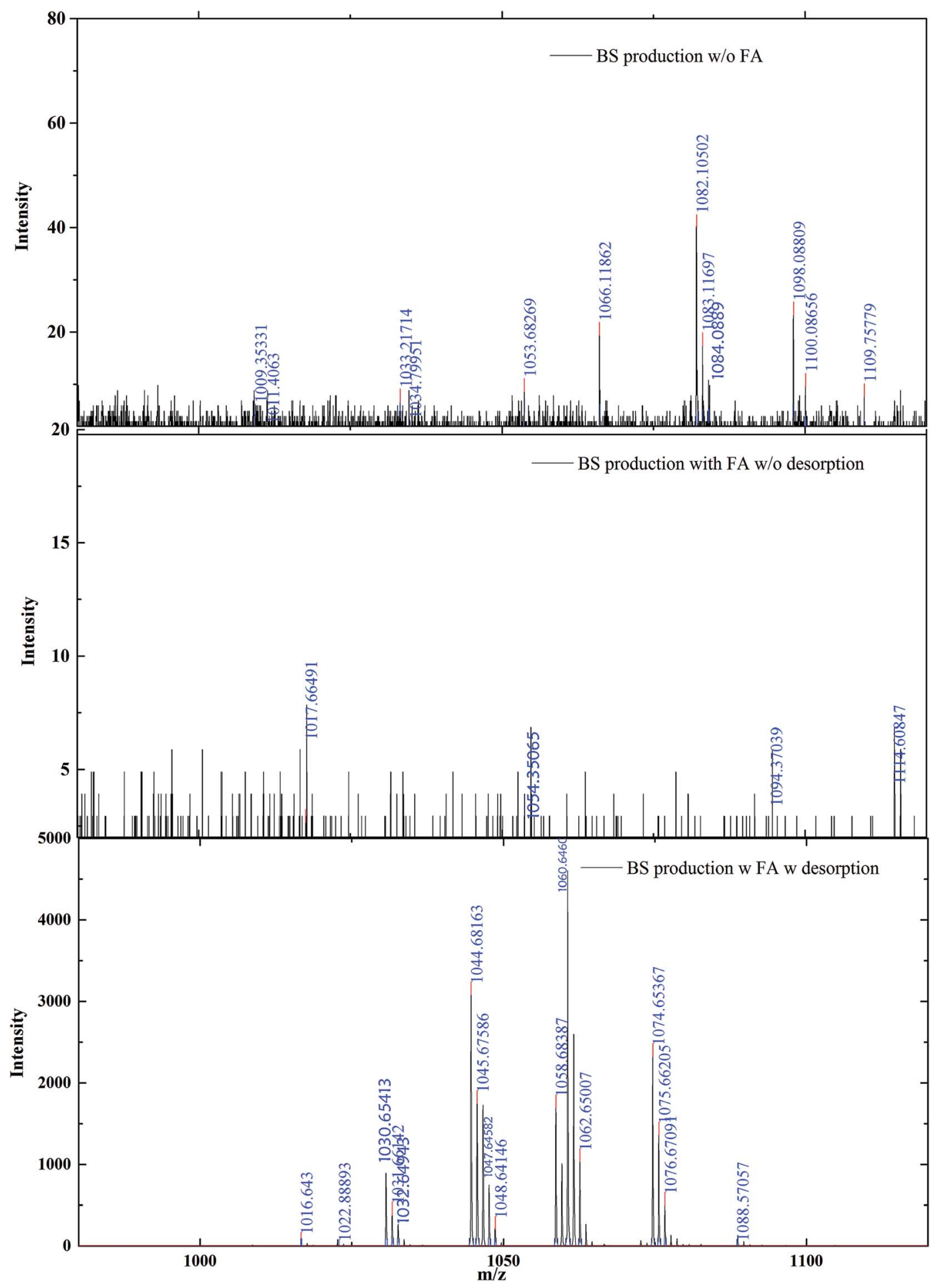

Fig. 4 Characterization of biosurfactant with MALDI-TOF.

carrier, such as $\alpha$-cyclodextrin, filter paper, and silica gel..$^{22} \mathrm{~A}$ mechanism for FA-enhanced biosurfactant production through the self-produced biocatalyst is proposed in Fig. 1(a). Biofilms, acting as a biocatalyst in this study, are microbial communities encased in a layer of self-produced matrix EPS and adhered to FA surfaces. As proposed in Fig. 1(a), those free-floating biosurfactant producers started to flow into the channels of the FA particles and initially attached to their surface within the first few hours. Those pioneers then quickly anchored themselves to the matrix via the production of pili, fimbriae, and exopolysaccharides. ${ }^{22}$ Following initial attachment, the proliferation and building of microcolonies on FA surface spontaneously occurred through the production of an extracellular matrix. The pore structure and surface charge of FA were major contributors 
a)

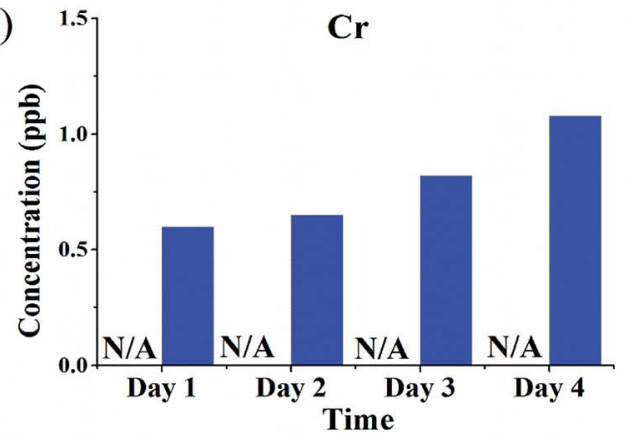

c)

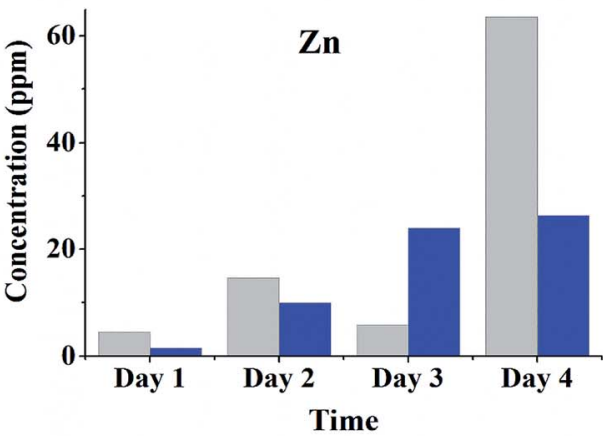

b)

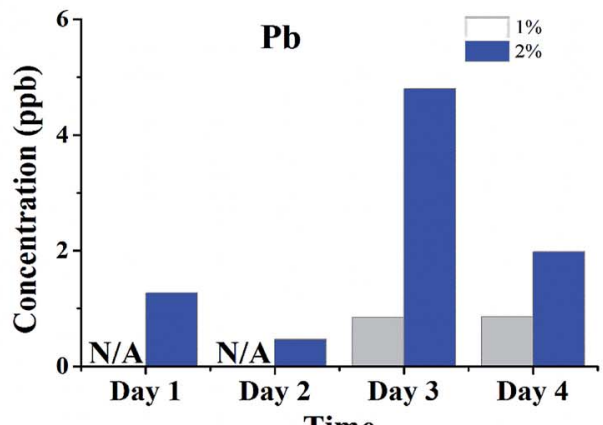

d)

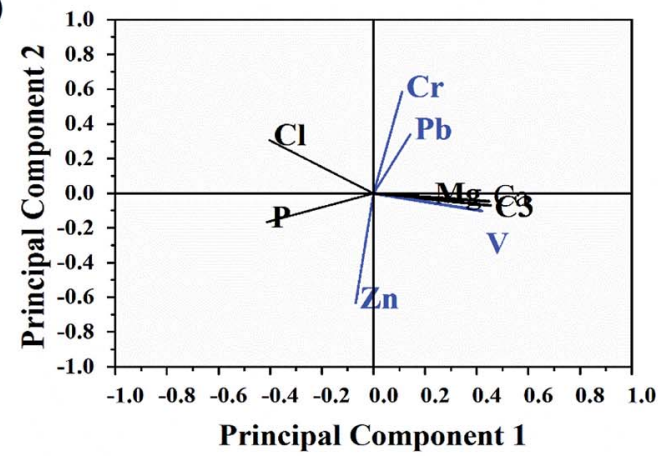

Fig. 5 (a-c) Bioleaching of heavy metal from FA surface; (d) principal component analysis of leachate metals.

to the bacterial adsorption process. ${ }^{23}$ Visual, elemental and spectroscopic analyses were carried out to provide multidisciplinary evidence with SEM and FTIR about the microstructure and surface chemistry of FA before and after the incubation process. The results are presented in Fig. 1(b). The SEM image of raw CBPP FA demonstrated that it consisted of a highly porous, platelet and fiber-shaped structure. Its energy dispersion X-ray spectroscopy (EDX) analysis revealed a dominant amount of carbon (C) on the surface. After incubation, a layer of biofilm was identified on the FA surface from the SEM image. The EDX analysis proved the growth of biofilm on the surface of FA. The dominant components on the surface of FA were found to be $\mathrm{P}, \mathrm{O}, \mathrm{C}$, and $\mathrm{Mg}$, the major components of biofilm. Surface properties play an important role in initial cell attachment. ${ }^{24}$ The rough surface of FA, which Fig. 1(b) presents, has been considered to be an excellent solid carrier to promote cell settlement and biofilm growth on its surface, owing to the enhanced cell-surface interactions and strengthened protection from shear force. ${ }^{25}$ The absorbent properties of FA offered the biosurfactant producer better access to localized nutrients, which thus have higher metabolic activity than free-living ones. Recent research has confirmed that microbes tend to reside in biofilms, rather than as free-floating forms ${ }^{26}$ Biofilm was able to provide biosurfactant producers with a stable environment under external stress (e.g. disinfectants and antibiotics) by reducing the diffusion of those compounds, ${ }^{27}$ thereby promoting their growth rate, and follow-up biosurfactant production.

The FTIR spectra (Fig. 1(b)) provided qualitative characterization of the surface of FA, primarily by providing information about functional groups. The major peaks acquired from raw FA material were in agreement with those reported by Martins et al. ${ }^{28}$ The carbonate $\left(870-1400 \mathrm{~cm}^{-1}\right)$ group was recognized in this study. Its presence in wood-based FA has been widely acknowledged, as the combustion process mineralizes the organic compounds, and transforms the basic cations to their oxide forms. They are later hydrated and subsequently converted into the forms of carbonates and phosphates. ${ }^{29}$ The alkane C-H bond stretch $\left(2700-3000 \mathrm{~cm}^{-1}\right)$ and carboxylic and/ or hydroxyl groups $\left(3200-3600 \mathrm{~cm}^{-1}\right)$ were identified from the FTIR analysis. ${ }^{30}$ The presence of $\mathrm{Al}$ and $\mathrm{Fe}$ as oxygen functional groups within $\mathrm{Al}-\mathrm{OH}$ and $\mathrm{Fe}-\mathrm{OH}\left(800-900 \mathrm{~cm}^{-1}\right)$ can be confirmed from Putra et $a l^{31}$ The FTIR spectrum of the FA samples after the incubation process is also presented in Fig. 1(b). This result showed an increased intensity of carboxylic and/or hydroxyl groups in $3200-3600 \mathrm{~cm}^{-1}$ and $800-1000 \mathrm{~cm}^{-1}$, verifying the existence of biofilm on its surface. It is recognized that biofilm is composed of up to $90 \%$ of water, ${ }^{32}$ while the rest consists of polysaccharide, protein, DNA, etc. The abundant carboxylic in FA and the biofilm surface may form a chemical bond structure with carbonate groups on the FA surface, thus leading to the disappearance of the stretching band at $1450 \mathrm{~cm}^{-1}$.

Fig. 1(c) provides biosurfactant productivity with the addition of FA. A rocketing biosurfactant production rate was observed after incubation for 18 hours. The assembled biocatalyst on FA particles accelerated the reaction by well over tenfold. Biosurfactant concentration increased from 9 CMD in the control sample to $100 \mathrm{CMD}$ in the FA sample after incubation for 24 hours. It is believed that biosurfactant production was stimulated through the quorum sensing system at this stage (Fig. 1(a)), not only to enhance the swarming motility of the biosurfactant producer, but also to alter the wettability and potential of the platform surface to facilitate their 
residence. ${ }^{22,33,34}$ The role of the lipopeptide biosurfactant as signaling molecules triggering robust biofilm formation for Bacillus strains under laboratory conditions has been identified. ${ }^{35}$ The intercellular communications within a biofilm further stimulated the up-and-down regulation of gene expression, enabling temporal adaptations, such as phenotypic variation and the ability to survive in nutrient-deficient conditions, ${ }^{36}$ thereby promoting the biosurfactant production. This study demonstrated the successful application of FA as a platform to stimulate the biosynthesis of lipopeptide through biofilm-encased cells and the finding in this study was comparable to that generated by Wigneswaran et al. ${ }^{37}$ This result further proved the enhanced production mechanism described in Fig. 1(a). Acting as molecular signal, biosurfactant was initially secreted to stimulate EPS production and biofilm formation at a relatively slow rate. ${ }^{35}$

It is acknowledged that cell attachment and biofilm formation will alter the physiochemical properties of a porous medium. The surface electrostatic charge of a porous medium will accordingly be affected by the attached biosurfactant production cells and the EPS matrix. Zeta potential measurement has been widely used to characterize the solid-liquid interface, obtaining the nature and charge information of a solid surface, and exhibiting the electrokinetic behavior of the solid-liquid interface. Therefore, the variation in zeta potential was investigated to shed light on the attachment of microbes and the growth of biofilm on the FA surface. The results are listed in Table 2. Zeta potentials of all FA particles were less than zero in the provided neutral buffer solution. Those with $2 \%$ FA dosage had the lowest starting zeta potential value, followed by $1 \%$ and then $0.5 \%$. This negative zeta potential might due to the initial conditioning process, resulting from the attraction of mineral groups in the growth medium, such as $\mathrm{PO}_{4}{ }^{3-}$ or $\mathrm{SO}_{4}{ }^{2-} .^{38} \mathrm{~A}$ sharp decrease in zeta potential was identified during the first six hours, confirming the strong attachment of negatively charged biosurfactant producer to the FA surface. The hydrophobic FA surface tended to enhance bacterial attachment onto its surface by removing adsorbed surface water, and attracting bacteria with hydrophobic properties. ${ }^{39}$ Microbes then embedded into a self-produced extracellular matrix. When biofilm was gradually produced, its major component, namely neutral polysaccharide, shielded and/or neutralized the negatively charged surface functional groups, such as DNA and protein, thereby slowing the decrease in zeta potential. ${ }^{12}$ This explained the relatively stable zeta potential during the next few hours. The higher the concentration of FA particles, the longer the biosurfactant producer took to finish biofilm assembly. After that, a continuous decrease in zeta potential was observed due to the production and adsorption of produced biosurfactants on FA particles. At pH 7.4, most functional groups at the hydrophilic moiety of the produced anionic lipopeptide biosurfactant molecule were protonated or compensated by a counter ion, leaving limited acidic residues (e.g., Glu and Asp) working as effective negatively-charged carriers. ${ }^{40}$ The continuous accumulation of those produced anionic lipopeptide biosurfactants thus decreased the zeta potential of the FA surface.

\subsection{Effect of FA dosage on biosurfactant production}

The effect of supplemental FA as a platform for stimulating the growth of biocatalyst upon the enhancement of biosurfactant production was assessed. The medium was supplemented by a fixed amount (i.e., $0.5 \%, 1 \%$ or $2 \%$ ) of FA carrier. The biosurfactant production rate was obtained using the selected Bacillus strain, and the correlation between FA dosage and biosurfactant production rate was evaluated (Fig. 2). The results clearly demonstrated the remarkable advantage of using FA to promote biosurfactant production. As Fig. 2(a) shows, the lag phase of biosurfactant production was clearly affected by FA dosage. Acceleration of production firstly took place in incubation samples with $0.5 \%$ FA dosage and was last found in the samples with $2 \%$ dosage. After 24 hours, biosurfactant production was increased from 9 CMD to 110, 100 and 70 CMD, respectively. Afterwards, a reduction in biosurfactant production was recognized, accompanied by a reduction in $\mathrm{pH}$ (Fig. 2(b)), which was due to the generation of secondary acid metabolites, such as uronic acid, when using sugar as the carbon source. ${ }^{4}$ Biosurfactant was produced and led to a reduction in surface tension (Fig. 2(c)).

The reduction in biosurfactant concentration in culture media may be attributed to the depletion of the carbon source, as Yeh et al. ${ }^{22}$ suggested. Produced surfactin might be assimilated as a carbon source for additional cell growth when another carbon source was not available. Dubey et al., ${ }^{\mathbf{1 6}}$ however, claimed that activated carbon could also be used as an absorbent to recover biosurfactants, thus contributing to the reduction in biosurfactant in the culture broth. In this study, biosurfactant product in the sediment was further separated from the fly ash through acetone desorption (Fig. 2(d)). The biosurfactant concentration in culture broth $(0.5 \%$ L, 1\% L, $2 \%$ L) and in FA particles $(0.5 \% \mathrm{D}, 1 \% \mathrm{D}, 2 \% \mathrm{D})$ are shown in Fig. 2(e). The adsorption of biosurfactant on FA particles gradually increased until the maximum adsorption capacity of FA was reached on the fifth day. The addition of $2 \%$ FA gave the highest final biosurfactant yield, namely 305 CMD. The yields with the addition of $1 \% \mathrm{FA}$ and $0.5 \%$ FA were $255 \mathrm{CMD}$ and 170 CMD, respectively. A positive relationship between FA dosage and biosurfactant production yield was observed. A higher dosage of FA provided a larger surface area of biocatalyst. Through serving as a cell growth booster for the growth of $B$. subtilis in the media, a higher biosurfactant production yield was achieved. In addition, a higher FA dosage led to a higher content of iron inside (Table 1), which also contributed to the increase in biosurfactant production yield. ${ }^{41}$

\subsection{Characterization of the biosurfactant product}

The surface activity properties of the generated biosurfactant product were characterized in this study by measuring the ST and CMC values. The results indicated that biosurfactant products generated by both FA-based medium and control sample could reduce the ST of water from 75 to $27.8 \mathrm{mN} \mathrm{m}^{-1}$. The CMC value of the biosurfactant generated by FA-based medium was $0.407 \mathrm{~g} \mathrm{~L}^{-1}$, lower than that generated by the control $\left(0.524 \mathrm{~g} \mathrm{~L}^{-1}\right)$. It was assumed that the attachment of 
biosurfactant production cells on the FA surface eased the purification process. Biosurfactant was more easily desorbed from FA particles than other impurities. Its purity was therefore enhanced. ${ }^{\mathbf{1 6}}$

FTIR was further examined in this study to acquire information about the chemical bonds (functional groups) of generated biosurfactant product. Fig. 3 presents the FTIR spectra of the biosurfactant products generated by FA-based medium and control samples in the region of $400-4500 \mathrm{~cm}^{-1}$. Both products had similar spectra, indicating that they share the same functional groups. The characteristic absorbance peaks at $700-950 \mathrm{~cm}^{-1}$ (peaks 1, 2, and 3) revealed the presence of an aliphatic long fatty acid chain. The stretching mode identified from the band range $1000-1250 \mathrm{~cm}^{-1}$ was evidence for the presence of carbonyl (peak 6) and amide groups (amide I band) (peaks 4 and 5). The peaks corresponded to the linkage group between the amine and carboxylic groups of the amino acids and to the carboxylic group of the fatty acid. ${ }^{42}$ FTIR results displayed absorbance in the range of 1600 to $1700 \mathrm{~cm}^{-1}$ (peaks 9 and 10), due to the deformation mode of the $\mathrm{N}-\mathrm{H}$ bond combined with the $\mathrm{C}-\mathrm{N}$ stretching mode, indicating the existence of an amide II band. A typical $\mathrm{C}-\mathrm{H}$ stretching vibration in the alkyl chain was identified from the band range $2700-2900 \mathrm{~cm}^{-1}$ (peaks 12 and 13). The FTIR results confirmed that the biosurfactant was lipopeptide in nature.

The structure of the lipopeptide biosurfactant was elucidated based on MALDI-TOF spectral analysis and the results (Fig. 4) are in accordance with those generated by Yang et al. ${ }^{\mathbf{4 3}}$ This study compared the biosurfactant products generated in an FA-based culture medium with and without a desorption process, and those generated in the control sample using FAfree growth medium. The results were in accordance with the CMD result generated in Section 3.2. Comparing the low intensity and limited identified biosurfactant product in FA-free culture broth, the FA-based medium showed higher productivity. The productivity of the biosurfactant product was increased almost 100 times after the desorption process. This result clearly indicated the existence of three groups of lipopeptide biosurfactants: namely surfactin $(\mathrm{m} / \mathrm{z} 1008,1016$, 1030, 1044, 1058, and 1060), itruin (1026, 1043, 1065, 1079, 1093), and fengycin $(1463,1485,1499,1587)$.

\subsection{Bioleaching of heavy metals from FA}

The leachability of heavy metals from FA by the biosurfactant producer is illustrated in Fig. 5(a)-(c), and the principal component analyses of the behaviors of the elements contained in FA are illustrated in Fig. 5(d). The behavior in the culture medium can be attributed to two groups. One group was bacterial growth-related elements, such as $\mathrm{Cl}, \mathrm{P}, \mathrm{Mg}, \mathrm{Ca}$, and $\mathrm{Al}$, while the other was heavy metals such as $\mathrm{Cr}, \mathrm{Pb}$ and $\mathrm{Zn}$. Increased leachability was identified for all three heavy metals ( $\mathrm{Cr}, \mathrm{Pb}$ and $\mathrm{Zn}$ ). Previous research revealed that the $\mathrm{Zn}$ extraction process was faster than the others. ${ }^{\mathbf{4 4}}$ This trend was also proved in this study. The slow extraction process for $\mathrm{Pb}$ and $\mathrm{Cr}$ might be due to the relatively high $\mathrm{pH}$ value in the growth medium, as bio-acidic dissolution was preferred in the bioleaching process. The bioleaching attempt in this study indicated that FA could be detoxicated after several runs of the incubation process and ease its disposal and treatment process. ${ }^{45}$

\section{Conclusion}

This study examined an environmentally-friendly and costeffective way to produce biosurfactant through applying a selfproduced biocatalyst immobilized on FA surface. The results indicated that the addition of FA particles at $2 \% \mathrm{w} / \mathrm{v}$ ratio triggered the growth of biofilm, thus remarkably increasing the biosurfactant production rate. The application of FA further enhanced biosurfactant purity, resulting in a lower CMC value. FTIR and MALDI-TOF characterized the product as a lipopeptide. The findings improved the understanding of the cultivation setups and shed light on the application of a fixedbed biofilm reactor for catalyzing bioproduct generation.

\section{Conflicts of interest}

The authors have no conflicts of interest to declare.

\section{Acknowledgements}

The authors would like to express their gratitude to the Natural Science and Engineering Research Council of Canada (NSERC), the Canada Foundation for Innovation (CFI), Canada Research Chairs (CRC) Program and Fisheries and Oceans Canada (DFO) for their support.

\section{References}

1 S. Shekhar, A. Sundaramanickam and T. Balasubramanian, Crit. Rev. Environ. Sci. Technol., 2015, 45, 1522-1554.

2 P. Jimenez-Penalver, M. Castillejos, A. Koh, R. Gross, A. Sanchez, X. Font and T. Gea, J. Cleaner Prod., 2018, 172, 2735-2747.

3 F. Fan, B. Zhang, P. L. Morrill and T. Husain, RSC Adv., 2018, 8(47), 26596-26609.

4 Z. Zhu, B. Zhang, B. Chen, Q. Cai and W. Lin, Water, Air, Soil Pollut., 2016, 227, 328.

5 X. Li, F. Fan, B. Zhang, K. Zhang and B. Chen, Int. Biodeterior. Biodegrad., 2018, 132, 216-225.

6 C.-Y. Chen and J.-S. Chang, Process Biochem., 2006, 41, 20412049.

7 Y. Zhi, Q. Wu and Y. Xu, Sci. Rep., 2017, 7, 40976.

8 C. Kokare, S. Chakraborty, A. Khopade and K. R. Mahadik, Indian J. Biotechnol., 2009, 8(2), 159-168.

9 R. Karande, L. Debor, D. Salamanca, F. Bogdahn, K. H. Engesser, K. Buehler and A. Schmid, Biotechnol. Bioeng., 2016, 113, 52-61.

10 B. Zhou, G. J. Martin and N. B. Pamment, Biotechnol. Bioeng., 2008, 100, 627-633.

11 B. Halan, K. Buehler and A. Schmid, Trends Biotechnol., 2012, 30, 453-465. 
12 Y. Liu and J. Li, Environ. Sci. Technol., 2007, 42, 443-449.

13 D. A. A. El-Fattah, W. E. Eweda, M. S. Zayed and M. K. Hassanein, Annals of Agricultural Sciences, 2013, 58, 111-118.

14 F. B. Rebah, R. Tyagi and D. Prevost, Bioresour. Technol., 2002, 83, 145-151.

15 Q. Cai, B. Zhang, B. Chen, Z. Zhu, W. Lin and T. Cao, Mar. Pollut. Bull., 2014, 86, 402-410.

16 K. V. Dubey, A. A. Juwarkar and S. Singh, Biotechnol. Prog., 2005, 21, 860-867.

17 J. D. Sheppard and C. N. Mulligan, Appl. Microbiol. Biotechnol., 1987, 27, 110-116.

18 M. Shavandi, G. Mohebali, A. Haddadi, H. Shakarami and A. Nuhi, Colloids Surf., B, 2011, 82, 477-482.

19 Q. Cai, Z. Zhu, B. Chen and B. Zhang, Water Res., 2019, 149, 292-301.

20 A. M. Akgün, Citeseer, 2005.

21 L. Li, S. Liu and J. Liu, J. Hazard. Mater., 2011, 192, 683-690.

22 M. S. Yeh, Y. H. Wei and J. S. Chang, Biotechnol. Prog., 2005, 21, 1329-1334.

23 O. Rendueles and J. M. Ghigo, FEMS Microbiol. Rev., 2012, 36, 972-989.

24 C. Goller and T. Romeo, in Bacterial Biofilms, Springer, 2008, pp. 37-66.

25 X. Z. Li, B. Hauer and B. Rosche, Appl. Microbiol. Biotechnol., 2007, 76, 1255-1262.

26 M. R. Frederick, C. Kuttler, B. A. Hense and H. J. Eberl, Theor. Biol. Med. Modell., 2011, 8, 8.

27 M. Berlanga and R. Guerrero, Microb. Cell Fact., 2016, 15, 165.

28 F. M. Martins, J. M. Martins, L. C. Ferracin and C. J. da Cunha, J. Hazard. Mater., 2007, 147, 610-617.

29 A. Demeyer, J. V. Nkana and M. Verloo, Bioresour. Technol., 2001, 77, 287-295.

30 W. J. Weber, J. Tang and Q. Huang, Environ. Sci. Technol., 2006, 40, 1650-1656.
31 E. K. Putra, R. Pranowo, J. Sunarso, N. Indraswati and S. Ismadji, Water Res., 2009, 43, 2419-2430.

32 J. Schmitt and H.-C. Flemming, Water Sci. Technol., 1999, 39, 77-82.

33 I. A. Ribeiro, M. R. Bronze, M. F. Castro and M. H. Ribeiro, J. Chromatogr. B: Anal. Technol. Biomed. Life Sci., 2012, 899, 7280.

34 S. Gélis-Jeanvoine, A. Canette, M. Gohar, T. Caradec, C. Lemy, M. Gominet, P. Jacques, D. Lereclus and L. Slamti, Research in Microbiology, 2016.

35 H. Zeriouh, A. Vicente, A. Pérez-García and D. Romero, Environ. Microbiol., 2014, 16, 2196-2211.

36 T. R. Garrett, M. Bhakoo and Z. Zhang, Prog. Nat. Sci., 2008, 18, 1049-1056.

37 V. Wigneswaran, K. F. Nielsen, C. Sternberg, P. R. Jensen, A. Folkesson and L. Jelsbak, Microb. Cell Fact., 2016, 15, 181. 38 F. Julien, M. Baudu and M. Mazet, Water Res., 1998, 32, 3414-3424.

39 M. Van Loosdrecht, J. Lyklema, W. Norde, G. Schraa and A. Zehnder, Appl. Environ. Microbiol., 1987, 53, 1893-1897.

40 H. Y. Fan, M. Nazari, G. Raval, Z. Khan, H. Patel and H. Heerklotz, Biochim. Biophys. Acta, Biomembr., 2014, 1838, 2306-2312.

41 Y. H. Wei, L. F. Wang and J. S. Chang, Biotechnol. Prog., 2004, 20, 979-983.

42 A. Gordillo and M. C. Maldonado, Chromatogr. Its Appl., 2012, 11, 201-225.

43 H. Yang, X. Li, X. Li, H. Yu and Z. Shen, Anal. Bioanal. Chem., 2015, 407, 2529-2542.

44 B. Xin, W. Jiang, X. Li, K. Zhang, C. Liu, R. Wang and Y. Wang, Bioresour. Technol., 2012, 112, 186-192.

45 X. Zeng, S. Wei, L. Sun, D. A. Jacques, J. Tang, M. Lian, Z. Ji, J. Wang, J. Zhu and Z. Xu, J. Soils Sediments, 2015, 15, 10291038. 